Itinéraires Itinéraires

Littérature, textes, cultures

2012-3 | 2013

Lire les villes marocaines

\title{
Bertrand Westphal, La Géocritique. Réel, fiction, espace
}

Paris, Minuit, 2007, 278 pages.

\section{Khalid Zekri}

\section{OpenEdition}

\section{Journals}

Édition électronique

URL : http://journals.openedition.org/itineraires/1024

ISSN : 2427-920X

Éditeur

Pléiade

\section{Édition imprimée}

Date de publication : 1 juillet 2013

Pagination : 169-173

ISBN : 978-2-343-01183-7

ISSN : $2100-1340$

\section{Référence électronique}

Khalid Zekri, « Bertrand Westphal, La Géocritique. Réel, fiction, espace », Itinéraires [En ligne], 2012-3 |

2013, mis en ligne le 01 décembre 2012, consulté le 22 septembre 2020. URL : http://

journals.openedition.org/itineraires/1024

Ce document a été généré automatiquement le 22 septembre 2020

\section{(c) $(1)$}

Itinéraires est mis à disposition selon les termes de la licence Creative Commons Attribution - Pas d'Utilisation Commerciale - Pas de Modification 4.0 International. 


\title{
Bertrand Westphal, La Géocritique. Réel, fiction, espace
}

Paris, Minuit, 2007, 278 pages.

\author{
Khalid Zekri
}

\section{RÉFÉRENCE}

Bertrand Westphal, La Géocritique. Réel, fiction, espace, Paris, Minuit, 2007, 278 pages.

1 Selon Bertrand Westphal, la géocritique nous renseigne sur le rapport que les individus entretiennent avec les espaces dans lesquels ils vivent et se meuvent. Elle permet d'opérer un décentrement des analyses spatiales qui, en règle générale, sont égocentrées dans la mesure où elles s'articulent autour du point de vue des personnages ou de l'auteur. C'est ce qui distingue par ailleurs la géocritique de l'imagologie. Tout en s'inscrivant dans une démarche globalement égocentrée, l'imagologie accorde une place importante au réalème (en tant que marqueur d'une référentialité extratextuelle) dans l'examen des représentations d'un espace donné chez un ou plusieurs auteurs. C'est, par ailleurs, ce réalème qui oriente les représentations que l'imagologie examine. Celle-ci est intéressante par rapport à l'analyse des espaces littéraires de l'ailleurs à travers l'étude des représentations de l'étranger dans la littérature ${ }^{1}$. De plus, l'interdisciplinarité est une condition requise dans ce type de démarches analytiques. C'est pour cela que l'altérité, en tant que rapport différentiel dans lequel s'opère une interaction entre le je et l'autre l'ici et l' ailleurs, constitue un élément nodal dans les études imagologiques. Ces études, en s'intéressant à l'écart entre regardant et regardé, ne peuvent faire l'économie de l'espace dans lequel s'opère cette interaction. L'espace relève ici non pas d'une simple image formée sur l'ailleurs regardé, mais d'une "hétéro-image» résultant de l'expérience de l'altérité. L'imagologie s'intéresse à la représentation de ces images à travers «l'imagination productrice » qui re-crée « littérairement l'étranger ${ }^{2}$ ». Le lien entre espace littéraire et espace de référence est ainsi évincé. La géocritique est, quant 
à elle, géocentrée dans la mesure où « elle place le lieu au centre des débats » (p. 185). L'intérêt du géocriticien est porté non pas sur les auteurs et leur rapport à tel ou tel lieu, mais sur le lieu lui-même tel qu'il apparaît, tel qu'il est représenté dans différents domaines artistiques (littérature, photographie, peinture, cinéma...). Si pour l'imagologie c'est le sujet représentant qui importe, pour la géocritique c'est le lieu représenté qui revient au centre de l'analyse. Dans cette optique, le lieu est analysé en relation avec son référent (extratextuel) car «le référent et sa représentation sont interdépendants, voire interactifs »(p.186).

2 La géocritique peut enrichir la théorie postcoloniale dans la mesure où elle n'analyse pas le lieu uniquement du point de vue du regardant, en l'occurrence le dominant. Elle confronte le lieu aux divers regards (internes et externes) qui le constituent en objet artistique étroitement lié au référent. Le regardant et le regardé sont ainsi pris en compte dans leurs différentes représentations d'un même lieu; lieu regardé à la fois par l'autre et par soi. Le lieu, confronté au regard de l'autre, se voit transformé en permanence dans le regard de celui qui, au quotidien, l'habite. Il en résulte une conception mobile du lieu car ce qui est intéressant c'est cet « espace commun, né au et du contact de différents points de vue" (p. 188). La multifocalisation sur un espace référentiel devient ainsi le mot clé car "dès l'instant où l'“écriture" du lieu est circonscrite à un seul auteur, [on s'écarte de la géocritique au sens strict du mot et] on rentre dans le cadre égocentré de l'analyse imagologique » (p. 192). La représentation n'est pas abordée par la géocritique à partir de son degré de vérissimilitude car la gradation, allant de l'élevé au bas en passant par le moyen, est peu pertinente.

La vocation de la géocritique est stratigraphique dans la mesure où elle reconstruit le lieu selon un principe archéologique. La re-présentation étant seconde présentation, la saisie du lieu se fait par rapport à «quelque chose » qui lui préexiste. Cela peut être un réalème (référentiel) ou un référent artistique (texte, image, film...). Dans ce cas, c'est la chaîne intertextuelle ou intericonique qui joue le rôle de référent esthétique. La multifocalisation prend trois formes modulables : endogène, exogène et allogène. Dans ces trois catégories, le point de vue reste relatif à la situation de celui qui regarde et observe l'espace de référence (p. 208). Ce rapport entre l'observateur, abstraction faite de son appartenance de genre, et l'espace va de l'intimité à l'extranéité en passant par la familiarité selon que le point de vue est endogène, exogène ou allogène. Soulignons cependant que la vision de l'espace, telle qu'elle émane du point de vue endogène, est surdéterminée souvent par l'autochtonie.

La géocritique s'inspire de la géographie sensorielle qui dénonce la prépondérance du visuel sur les autres foyers perceptifs. Ces géographes dressent une taxinomie sensorielle dans laquelle "l'odorat, le toucher et le goût seraient intimes, corporels, passifs tandis que la vue et l'ouïe seraient des sens distants, cérébraux » (p. 215). Ce qui intéresse la géocritique telle que la conçoit l'auteur, c'est la perception véhiculée par l'ensemble des sens qui reçoivent l'information et l'élaborent mentalement. En contribuant à l'orientation de l'individu dans l'espace, les sens acquièrent une valeur géographique. La polysensorialité exerce un impact important sur le sujet dans son procès de présentation du contexte dans lequel il se meut. L'étude du lieu est une question de synesthésie aussi bien individuelle que collective, c'est-à-dire une question d'intersubjectivité.

5 Cette démarche s'inscrit dans une optique hétérogène des points de vue car l'espace humain est une arborescence qui prend différentes orientations (p. 227). La perception 
d'une ville ne réside pas forcément dans son aspect ni dans sa superficie, mais plutôt dans sa chronotopie, entendue comme intrication indissoluble du temps et de l'espace. Bertrand Westphal, en s'inspirant de La Danse de la vie d'Edward T. Hall, redéfinit les notions opératoires de monochronie et de polychronie: «Est monochrone la vision (hégémonique) qui consiste à discerner dans le monde une seule temporalité commune à l'ensemble de l'humanité. Est, par conséquent, polychrone la vision qui assigne une temporalité différente à chaque aire culturelle » (p. 230).

6 À vrai dire, Bertrand Westphal ne résout pas vraiment le problème de la référentialité en incluant la représentation littéraire dans un réel élargi (p. 191). Cet argument peut paraître séduisant, mais il ne fait que contourner l'épineuse question du lien entre le texte littéraire et la réalité extralittéraire. Le problème que pose l'articulation de la littérature au réel, c'est celui de savoir si, dans une perspective géocritique, la création littéraire re-produit le référent spatial tel qu'il est dans le monde des expériences. Il est, bien entendu, presque un lieu commun de dire aujourd'hui que l'univers de la fiction n'est pas celui du réel. Mais, y a-t-il vraiment une autonomie de l'un par rapport à l'autre? C'est la question que pose Westphal, mais à laquelle il donne une réponse, certes claire, mais pas tout à fait convaincante. Dans son argumentation, il passe en effet du problème que pose le lien entre fiction et réalité à celui de la fiction en tant que forme de représentation qui circule dans le monde social. Cette manière de traiter le problème, même si elle tient compte de l'hétéroréférentialité, ne répond pas à la question initialement posée et, par conséquent, laisse en suspens l'analyse du lien entre fiction et référent. De plus, l'auteur tombe dans des généralisations, probablement à cause de son enfermement dans un corpus euroaméricain et sa méconnaissance des textes viatiques écrits par des auteurs orientaux. C'est en effet le cas quand il dit que «le texte était la propriété exclusive d'une société - la société occidentale [sic] - qui s'arrogeait le monopole du regard transcrit » (p. 201). Or, Il existe tout un corpus de relations de voyage arabes qui transcrit le regard des chrétiens et des musulmans d'Orient sur l'Europe, le Nouveau Monde et l'Extrême Orient.

7 De ce point de vue, et surtout par rapport à notre monde globalisé, les analyses d'Arjun Appadurai ${ }^{3}$ peuvent compléter les apports de la géocritique à travers l'analyse des paysages culturels. C'est pourquoi nous nous permettrons d'en donner ici un aperçu, même si l'ouvrage de Bertrand Westphal n'y fait pas allusion. La complexité des flux culturels globaux a conduit Appadurai à les répertorier en cinq dimensions: les ethnoscapes, les médiascapes, les technoscapes, les financescapes et les idéoscapes. Ces termes sont construits à partir du mot "Landscape » (paysage) et permettent de montrer les différentes facettes des paysages sociaux qui caractérisent la globalisation culturelle. Ces paysages, qui constituent des flux, sont les éléments constitutifs des mondes construits par des imaginaires qui ont leur historicité et qui sont liés à des groupes diasporiques. Ces paysages sont appelés «mondes imaginés » et c'est à travers eux que le matériel culturel traverse les frontières nationales.

8 L'ethnoscape est « le paysage formé par les individus qui constituent le monde mouvant dans lequel nous vivons : touristes, immigrants, réfugiés, exilés ${ }^{4}$ ». C'est un paysage d'identité de groupe. Il est caractérisé par la déterritorialisation et la reconfiguration des projets ethniques. La stabilité des communautés et les différentes formes d'affiliation traditionnelles existent toujours, mais elles sont traversées par ce flux de mouvement humain. Le technoscape est « la configuration globale et toujours fluide de la technologie, et le fait que cette dernière, haute ou basse, mécanique ou 
informationnelle, se déplace aujourd'hui à grande vitesse entre les frontières jusque-là infranchissables ${ }^{5}$ ». Les financescapes constituent le flux des capitaux et des finances et relèvent de «la disposition du capital mondial [qui] forme désormais un paysage plus mystérieux, plus rapide et plus difficile à suivre que jamais : les marchés de change, les bourses nationales et les spéculations sur les biens et les services font passer, à la vitesse de la lumière, des sommes colossales à travers les tourniquets nationaux [... $]^{6}$ ». Les médiascapes, « ce sont à la fois la distribution des moyens électroniques de produire et de disséminer de l'information (journaux, magazines, chaînes de télévisions et studios cinématographiques), désormais accessibles à un nombre croissant d'intérêts publics et privés à travers le monde, et les images du monde créées par ces médias ${ }^{7}$ ». Les médiascapes fournissent des images et des récits d'identification dans lesquels interfèrent la valeur marchande, l'information et la politique. Elles sont ensuite dispensées à de nombreux publics disséminés dans différents pays et continents. Enfin, les idéoscapes sont constitués d'une succession d'images, "mais ils sont souvent directement politiques et en rapport avec les idéologies de l'État et les contreidéologies de mouvements explicitement orientés vers la prise du pouvoir de l'État ou d'une de ses parties ${ }^{8} »$. Ce qui est intéressant dans cette transversalité du regard sur le paysage humain face à la globalisation, c'est la remise en question du compromis ironique entre ce que les hommes pouvaient imaginer et ce que la vie sociale leur permet. La vie humaine n'est plus appréhendée comme un simple résultat du monde tel qu'il va, mais comme une tentative d'imaginer le quotidien, de manière incessante, en fonction du landscape avec lequel les individus entrent en interaction. Cela rend bien évidemment notre monde actuel à la fois rhizomatique et schizophrène car, tout en étant dans un raisonnement de proximité électronique, l'homme post-national développe en même temps des thèses sur le déracinement et l'aliénation de l'individu par rapport au groupe.

\section{NOTES}

1. Cf. Jean-Marc Moura, L'Europe littéraire et l'Ailleurs, Paris, PUF, 1998, p. 35.

2. Ibid., p. 45.

3. Arjun Appadurai, né à Bombay en 1949, est un sociologue culturaliste et un anthropologue, auteur de nombreux ouvrages, notamment en traduction française : Après la colonisation. Les conséquences culturelles de la globalisation (Payot, 2005); Géographie de la colère. La violence à l'âge de la globalisation (Payot, 2009).

4. Arjun Appadurai, Après la colonisation. Les conséquences culturelles de la globalisation, Payot, 2005, p. 71.

5. Ibid., p. 72.

6. Ibid., p. 73.

7. Ibid., p. 73-74.

8. Ibid., p. 74. 


\section{AUTEURS}

\section{KHALID ZEKRI}

Université Moulay Ismaïl - Meknès

Équipe d'études culturelles et postcoloniale 